\title{
CIUDADANÍA MULTINIVEL Y SU ENCAJE CONSTITUCIONAL
}

\author{
Multilevel citizenship and ownership
}

\author{
Clara Souto Galván \\ Profesora contratada doctora de Derecho Constitucional \\ Universidad Rey Juan Carlos
}

http://dx.doi.org/10.18543/ed-68(1)-2020pp449-473

Recibido: 24.03.2020

Aceptado: 12.06 .2020

\section{Resumen}

La ciudadanía conlleva el reconocimiento de unos derechos fundamentales y constitucionales a los que está sujeto por el país en el que vive, pero ¿quién es considerado ciudadano? ¿quién es titular del reconocimiento de esos derechos en un país? Preguntas que no siempre obtienen una fácil respuesta, sobre todo porque la ciudadanía ha alcanzado una dimensión multinivel que dificulta dicho reconocimiento, comprobando que será dependiendo del país de origen o del país de residencia, si dicho país pertenece a la Unión Europea o son nacionales de terceros países, dependiendo a su vez de los tratados firmados con esos países, todo ello aspectos a los que se intenta dar respuesta, desde el punto de vista legislativo y constitucional, detallando los avances más significativos que se han desarrollado a nivel Europeo, estatal y autonómico, respecto de las directivas, convenios y acuerdos internacionales y reformas constitucionales, como por ejemplo la reforma del art. 13.2 de la Constitución española, que además de ser la primera reforma constitucional supuso un avance importante en el reconocimiento de derechos políticos de los extranjeros en las elecciones municipales, pues se les atribuía el derecho de ser electo, aunque podría ser revisada para alcanzar un mayor grado de igualdad los nacionales de un país con los residentes del mismo.

En conclusión, lo que se propone es democratizar el acceso a la nacionalidad cuando se trate de una condición para el disfrute de la ciudadanía, reduciendo plazos y requisitos, pero también evitando el reconocimiento de dichos derechos solo por 
adquisición de la nacionalidad, sino que los residentes de larga duración, sin dicho requisito puedan ser sujetos de reconocimiento de derechos, en concreto del derecho de sufragio activo y pasivo. Para lo que sería necesario modificar de nuevo el art. 13.2 incluyendo a los residentes de larga duración y eliminando la necesidad de basarse en el principio de reciprocidad, lo que permitiría una mayor integración de los nacionales de otros países en el Estado de residencia.

\section{Palabras clave}

Ciudadanía, nacionalidad, multinivel, derechos, libertades.

\section{Abstract}

Citizenship involves the recognition of fundamental and constitutional rights to which they are subject by the country in which they live, but who is considered a citizen? Who owns the recognition of those rights in a country? Questions that do not always get an easy answer, especially because the citizens have reached a multilevel dimension that hinders this recognition, proving that it will depend on the country of origin or the country of residence, if that country belongs to the European Union or are nationals of third countries, depending in turn on the treaties signed with these countries, all of which aspects are intended to be answered, from the legislative and constitutional point of view, detailing the most significant advances that have been developed at European, State and autonomic, with respect to directives, conventions and international agreements and constitutional reforms, such as the reform of art. 13.2 of the Spanish Constitution, which in addition to being the first constitutional reform represented an important advance in the recognition of political rights of foreigners in municipal elections, as they were given the right to be elected, although it could be revised to achieve greater degree of equality the nationals of a country with its residents.

In conclusion, what is proposed is to democratize access to nationality when it is a condition for the enjoyment of citizenship, reducing time and requirements, but also avoiding the recognition of those rights only by acquisition of nationality, but rather long-term residents, without this requirement may be subject to recognition of rights, in particular the right to active and passive suffrage. For which it would be necessary to modify again the art. 13.2 including long-term residents and eliminating the need to rely on the principle of reciprocity, which would allow a greater integration of nationals of other countries in the State of residence.

\section{Key words}

Citizenship, nationality, multilevel, rights, freedoms. 
SUMARIO: I. CONSIDERACIONES PREVIAS. II. NACIONALIDAD Y CIUDADANÍA. III. CiUdADANÍA MUlTINIVEL. IV. TITUlaRIDAD DE LOS DERECHOS Y LIBERTADES. V. ¿ES NECESARIA UNA REFORMA CONSTITUCIONAL RESPECTO A LA CIUDADANÍA?. BIBLIOGRAFÍA.

\section{CONSIDERACIONES PREVIAS}

En el siglo XXI la noción de ciudadano no va enfocada exclusivamente a una serie de rasgos comunes de carácter cultural, sino al reconocimiento de unos derechos, no sólo fundamentales, sino también políticos y constitucionales a todos los miembros de un país. El problema se plantea sobre quiénes son las personas a las que se les reconocen estos derechos.

Partiendo del principio democrático se deberían reconocer por igual a todas las personas pertenecientes a un país y, como establece la Constitución española en su preámbulo «se debe establecer una sociedad democrática avanzada». ¿Pero cómo se materializa esto?

La Comisión europea define ciudadanía cívica como un conjunto de derechos y obligaciones básicas. En 2004 La Comisión por primera vez hacía referencia a este concepto, que en cierta manera garantizaba a los residentes de larga duración derechos y obligaciones, independientemente de que pudieran o no adquirir la nacionalidad del país de acogida.

La Comisión ha jugado un papel importante a la hora de promover nueva legislación en asuntos diversos, que van desde la no discriminación hasta la ciudadanía cívica. El objetivo es que los nacionales de terceros países pueden incluirse dentro de un concepto de ciudadanía de la Unión. La noción de ciudadanía cívica se inserta como una vía imprescindible para garantizar la integración de los inmigrantes ${ }^{1}$. Por lo tanto, se presenta como una categoría que tiene como principal función facilitar el establecimiento con éxito de los inmigrantes en la sociedad, y como un primer paso para la adquisición de la nacionalidad de un Estado miembro ${ }^{2}$.

De esta primera definición de ciudadanía cívica, se está procesando una nueva construcción jurídica, la ciudadanía multinivel ${ }^{3}$. Este reconocimiento

${ }^{1}$ Unión Europea. Comunicación de la Comisión al Parlamento europeo, al Consejo, al Comité económico y social europeo y al Comité de las Regiones, sobre inmigración, integración y empleo, Bruselas, COM. 3.6.2003, pp.32-33.

2 Zapata-Barrero, R. (2004). Una nueva «filosofía» de la UE: tradición versus innovación en la propuesta de ciudadanía cívica, II Seminario inmigración y Europa. Cinco años después de Tampere, Barcelona, Fundación Cibob, p.63.

3 Ver Moreno, L. (2012). «Ciudadanía multinivel», en Ciudadanía y derechos sociales (IV Congreso Internacional de derechos humanos), Vitoria-Gasteiz, Servicio central 
multinivel de la condición de ciudadano va parejo al desarrollo legislativo de cada país, en su progresiva adaptación de la legislación y la equiparación de condiciones de todos los individuos de un país, basado en el principio de igualdad imperante en todo Estado democrático, respetando los diversos grados de vinculación y sujeción al país.

El concepto de ciudadanía multinivel es más propio del Estado social y democrático de Derecho, porque desde el punto de vista democrático la nacionalidad y la ciudadanía han de construirse desde una sociedad de individuos libres e iguales que se relacionan entre sí, y no desde la pertenencia de una nacionalidad.

Para lo que será necesario adaptar las Constituciones, por ejemplo, en el caso de España, modificar los conceptos ordinarios, incluyendo el concepto multinivel desde la norma superior del ordenamiento jurídico, para poder incluir en el art.13.2 a los residentes, pues no se contemplan en ningún sitio, y pueden llegar a tener más vinculación con el país en el que reside, que por su nacionalidad.

Modificaciones que nos ayudarán a ir más allá del concepto de ciudadano europeo para conseguir una completa integración, rompiendo con los compromisos establecidos, y acabar con las fronteras.

Otra de las apuestas para la consecución de la integración es mediante la educación, desde Europa se está intentando dar prioridad al reconocimiento del derecho a la educación a todas las personas para lograr la consideración de ciudadanos, como pilar fundamental del reconocimiento de derechos. En este contexto, el objetivo general para mejorar la ciudadanía activa, la igualdad de oportunidades y la cohesión social, incluye aspectos como el acceso de los inmigrantes y de sus hijos a los sistemas de educación y formación ${ }^{4}$.

\section{NACIONALIDAD Y CIUDADANÍA}

La noción de ciudadanía desde un punto de vista histórico fue anterior al concepto de nacionalidad, ya se encontraba entre las premisas de Aristóteles que relacionaba la ciudadanía con la ciudad, matizando también la definición de ciudadano ${ }^{5}$. El término Nación y el término nacionalidad surgirán en la

de publicaciones del Gobierno Vasco, pp. 113-125.

${ }^{4}$ Unión Europea. Comunicación de la Comisión al Consejo, al Parlamento Europeo, al Comité Económico y Social Europeo y al Comité de las Regiones sobre inmigración, integración y empleo (2003), Comisión de las Comunidades Europeas, https://ec.europa. eu/transparency/regdoc/rep/1/2003/ES/1-2003-336-ES-F1-1.Pdf

5 «La asociación de muchos pueblos forma parte de una ciudad completa, que llega, si puede decirse así, a bastarse absolutamente a sí mismo, teniendo por origen las necesidades de la vida, y debiendo su subsistencia al hecho de ser éstas satisfechas», se hace un 
Revolución francesa. La Constitución de 1791 especifica que para adquirir la condición de ciudadano activo hace falta ser nacional. Este requisito no debería haberse mantenido en el desarrollo histórico, dejando atrás la idea liberal de ciudadanía, contraria a los principios de un Estado democrático, basados entre otros, en la igualdad, pues dicha condición no debería ser excluyente de la condición como ciudadano/a de un país, sobre todo, afectando dicha condición al pleno reconocimiento de los derechos de un país por el hecho de ser personas 6 .

Lo que nos puede llevar a la siguiente cuestión ¿sólo pueden ser ciudadanos los nacionales de un país? La condición de ciudadano dentro de un Estado deriva de la condición de persona libre e igual sujeta a su ordenamiento jurídico y no por la condición de nacional de ese Estado ${ }^{7}$. La condición de nacional puede ser una característica más del ciudadano, pero no puede ser un criterio de exclusión para ejercer la soberanía popular. ${ }^{8}$

El concepto de ciudadanía se solapa al de nacionalidad, Villaverde hace dicha apreciación al definirla como «un conjunto de reglas jurídicas que regulan el estatus de cada persona y el régimen jurídico que a él va aparejado en el seno de un conjunto del ordenamiento jurídico estatal o supraestatal, lo que se pretende es reflejar la idea de que se puede ser ciudadano de un Estado con diversos niveles de intensidad y estar sujetos a diversos sistemas jurídicos diferenciados sin dejar de ser ciudadano» ${ }^{9}$.

Hay otros aspectos que también se consideran imprescindibles del concepto de ciudadanía. Rodríguez Ruiz hace referencia a dos dimensiones diferenciadas de dicho concepto. Por un lado, al igual que Villaverde, identifica ciudadanía con nacionalidad y una segunda dimensión, en la que la define

análisis más profundo en Souto Galván, C. (2015), «Hacia un nuevo concepto de ciudadanía», Tur Ausina, R. (Dir.) Problemas actuales de derecho constitucional en un contexto de crisis, Comares, Granada.

${ }^{6}$ Márz, R. (2005). «Nacionalismos e inmigración en Francia: la Republique une et indivisible y el affaire du foulard», Revista de Estudios políticos (nueva época), nº 129 , Madrid, julio-septiembre, p.7.

7 Villaverde MenÉndeZ, I. (2012). «La ciudadanía borrosa: Ciudadanías multinivel», Cuadernos monográficos de teoría del estado, derecho público e historia constitucional, $\mathrm{n}^{\mathrm{o}}$ 7, Ejemplar dedicado a: El pueblo del Estado. Nacionalidad y Ciudadanía en el Estado Constitucional. Aláez Corral, B. (coord..), p. 296.

Biglino CAmpos, P. (1995). «Ciudadanía europea y legitimidad democrática», Revista de Estudios Europeos, n $^{\circ}$ 9, pp. 7-12. La profesora Biglino en este estudio desarrolla la idea de que el disfrute de los derechos políticos que componen el status de ciudadano exige dos requisitos más: el ejercicio del derecho de petición que suele reservarse a los mayores de edad mientras que para el derecho de voto no sólo se exige la nacionalidad y la mayoría de edad sino también la inscripción en el censo electoral.

9 Villaverde Menéndez, I. (2012). «La ciudadanía borrosa: Ciudadanías multinivel»,op. cit...p.292-293. 
como un estatus participativo ${ }^{10}$, lo que viene a colación con la importancia de la primera reforma constitucional del art. 13.2 $\mathrm{CE}$ y, con la propuesta de una nueva reforma constitucional sobre este aspecto, que permita igualar la participación política de las personas nacionales y residentes de un país, por lo que les permite a los titulares de estos derechos participar en la formación de la voluntad del Estado ${ }^{11}$.

En los últimos años, ha habido un aumento muy significativo de la población extranjera residente en España. En 2016 aumentó el número de extranjeros por primera vez desde 2011 en 6.892, aunque es importante señalar que en estos resultados influye el proceso de adquisición de nacionalidad española según los datos del INE. ${ }^{12}$ Los datos más recientes, aunque provisionales aún, son de junio de 2019, según los cuales la población extranjera de España aumentó en 276.186 personas durante 2018 y se situó en 46.934 .632 habitantes a 1 de enero de $2019^{13}$.

Los conceptos jurídicos de nacionalidad y ciudadanía siguen dos vías paralelas, pues son términos que se utilizan de manera sinónima, sin atender al aspecto más significativo: la ciudadanía, entendida como cualidad formal de la persona que le adscribe a un colectivo humano sobre el que ejerce su poder el Estado, y la nacionalidad, como proceso jurídico de integración del individuo en las diferentes esferas de participación social, y especialmente en la política ${ }^{14}$.

La nacionalidad y la ciudadanía suponen la forma de medir el mayor o menor grado de participación social y política, así como su grado de sujeción al ordenamiento jurídico y constitucional de un país. La nacionalidad, conlleva el vínculo jurídico que une a la persona con el Estado, además de ser un derecho fundamental, para la adquisición de la nacionalidad, es necesario como requisito, la residencia en el país de manera continuada durante 10 años, período inmediatamente anterior a la solicitud, aunque hay casos donde el período de residencia se puede reducir ${ }^{15}$.

10 Ver un análisis más profundo sobre este tema en Rodríguez Ruiz, B. (2016). «Las dos caras de la ciudadanía moderna: entre la nacionalidad y el estatus participativo», $R e$ vista Europea de Derechos fundamentales, primer semestre, pp. 17-18.

${ }^{11}$ Fraile Ortiz, M. (2003). El significado de la ciudadanía europea, Centro de Estudios Políticos y Constitucionales, Madrid, p. 34.

12 Recuperado de: $h$ ttp://www.ine.es/prensa/cp_2017_p.pdf

13 Recuperado de: http://www.ine.es/prensa/cp_e2019_p.pdf

14 Aláez CORRAL, B. (2005). «Nacionalidad y ciudadanía: una aproximación histórica-funcional», vol.6, Historia Constitucional, pp.88-89.

${ }^{15}$ Cinco años: para la concesión de la nacionalidad española a aquellas personas que hayan obtenido la condición de refugiado. Dos años: para los nacionales de países iberoamericanos, Andorra, Filipinas, Guinea Ecuatorial, Portugal o personas de origen sefardí. Un año: El que haya nacido en territorio español, el que no ejerció debidamente su derecho a adquirir la nacionalidad española por opción, el que haya estado sujeto legal- 
El Estado social y democrático de Derecho ha significado un avance en la relación inmigración-nacionalidad-ciudadanía. El principio democrático junto con la supremacía de la Constitución, respecto al resto del ordenamiento jurídico, ha permitido una consolidación de la figura del ciudadano, (aunque aún es necesario reformar la Constitución sobre este aspecto) afianzando el estatus jurídico del individuo mediante la adquisición de la nacionalidad o incluso su participación en los comicios locales sin necesidad de ser nacional (art. 13.2 CE). Es decir, asentar constitucionalmente la situación de personas extranjeras que residen en España de manera legal, pudiendo adquirir la nacionalidad y, por tanto, los derechos que de ella derivan, o bien, sin necesidad de ser nacional, participar plenamente como ciudadano en la vida política y jurídica de nuestro país.

Nacionalidad y ciudadanía son dos conceptos que se han unido tan nítidamente que se utilizan indistintamente en los países europeos, incluso llegando a absorber el término de ciudadanía al de nacionalidad ${ }^{16}$, entendiendo el concepto de ciudadanía como la capacidad de participación del individuo como miembro de pleno derecho de la comunidad estatal a la que está sometido ${ }^{17} \mathrm{y}$, el principio de nacionalidad, según Elisa Pérez Vera, sería el vínculo existente entre el Estado y el individuo, que permite identificarle como miembro de su población ${ }^{18}$.

Desde el punto de vista jurídico-constitucional, el punto de desencuentro entre nacionalidad y ciudadanía se situaría en el artículo 1.2 de la

mente a la tutela (bajo la vigilancia de un tutor), guarda o acogimiento (el acogimiento que permite la reducción de residencia legal a un año es aquél en que existe resolución de la entidad pública que tenga en cada territorio encomendada la protección de menores y los acogimientos que estén judicialmente reconocidos) de un ciudadano o institución españoles durante dos años consecutivos, incluso si continuare en esta situación en el momento de la solicitud, el que, en el momento de la solicitud, lleve un año casado con un español o española y no esté separado legalmente o de hecho, el viudo o viuda de española o español, si en el momento de la muerte del cónyuge no estaban separados, de hecho o judicialmente.

http://www.mjusticia.gob.es/cs/Satellite/Portal/es/ciudadanos/tramites-gestionespersonales/nacionalidad-residencia

${ }^{16}$ En el ámbito anglosajón, a veces, se contrapone la nacionalidad, entendida como ciudadanía formal a la ciudadanía en sentido estricto, entendida como ciudadanía material, y otras veces se utiliza el término ciudadanía como sinónimo de ambas categorías, en AláEz Corral, B. (2005). «Nacionalidad y ciudadanía: una aproximación históricafuncional», Historia Constitucional, vol. 6, p.89.

${ }_{17}$ Cfr. Aláez Corral, B. (2005). «Nacionalidad y ciudadanía: una aproximación histórica-funcional», op.cit., p. 89.

18 PÉrez Vera, E. (1996). «Citoyenneté de 1'Union Européenne, nationalité et condition des étrangers» Recuil des Courses. Collected curses of the Hague Academy of International Law, Vol. 261, Martinus Nifhoff Publishers, p. 278. 
Constitución española, es decir, sobre quién recae la soberanía: el sujeto nacional; por lo que, la distinción, en cierta manera, se daría en el tiempo. El nacional es titular de todos los derechos por el simple hecho de haber nacido en ese territorio, mientras que el extranjero adquirirá dicha condición cumpliendo determinados requisitos de tiempo de residencia. ¿Pero es necesario ser nacional para ser considerado ciudadano?

En esta cuestión es donde encontraríamos los diferentes niveles de ciudadanía:

- Ciudadanía relativa (pasiva): atributiva de la condición de representado políticamente, en tanto integrante de la nación o el pueblo del Estado, y de la garantía de los derechos civiles (de libertad) de la persona.

- Ciudadanía absoluta (activa): ligada a la atribución de los derechos políticos que correspondería a un círculo de sujetos más reducido de ciudadanos.

Pero dicha distinción, como aclara Aláez, no se sostiene en estos momentos, pues la primera de ellas se identifica con lo que hoy denominamos nacionalidad, mientras que la segunda corresponde al concepto moderno de ciudadanía.

Por lo que, podemos concluir, exponiendo que ambos conceptos se encuentran íntimamente ligados, que se utilizan indistintamente, pues, aunque se entienda que es necesario ser nacional para ser considerado ciudadano, como se planteó en el período revolucionario francés, con claras distinciones en comparación con el planteamiento del principio democrático, lo cierto es, que los extranjeros residentes de manera legal en España también serán considerados ciudadanos. Es cierto, no obstante, que existe un límite relativo al pleno reconocimiento de derechos, por lo que se entiende la ciudadanía desde diferentes niveles de participación política y social, como sujeto vinculado al Estado y a su ordenamiento jurídico y constitucional.

\section{III.CIUDADANÍA MULTINIVEL}

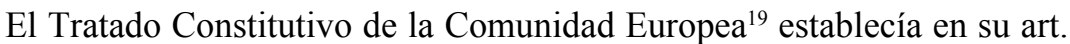
17 , que «será considerado ciudadano europeo toda persona que ostente la nacionalidad de algún país miembro». Por lo tanto, se unen dos caminos hasta entonces desconocidos, en primer lugar, reconocen que ser nacional de un Estado miembro de la UE conlleva la adquisición de un nuevo concepto,

${ }_{19}$ Vigencia desde 12 de junio de 1985. Esta revisión vigente desde 8 de noviembre de 2001 hasta 1 de diciembre de 20. 
la ciudadanía europea, lo que se suma una nueva escala al concepto de ciudadanía, adquiriendo un nuevo calificativo «europeo» ${ }^{20}$.

¿Qué consecuencias supone este nuevo reconocimiento? Por un lado, es un requisito ser nacional de un país miembro de la UE, por lo que este artículo ya supone una discriminación a aquellos extranjeros residentes de un país miembro, pues se les excluye de esta nueva ecuación, no podrán ser considerados ciudadanos europeos los no nacionales, es decir, la residencia en el territorio no ha logrado suplir al criterio de nacionalidad para ser ciudadano europeo $^{21}$.

En 1997 se optó por incorporar al texto del Tratado de Ámsterdam la aclaración «la ciudadanía de la Unión será complementaria y no sustitutiva de la ciudadanía nacional» aunque este texto sufrió una nueva modificación en el Tratado de Lisboa sustituyendo complementaria por adicional. Quedando recogido dicho texto en el TFUE y también en el TUE, en el que se declara que «la ciudadanía de la Unión se añade a la ciudadanía nacional sin sustituirla». Aunque ha habido diversas interpretaciones por los cambios que se produjeron en este artículo, lo que queda claro es que la ciudadanía nacional es la principal y la ciudadanía europea la complementa, pues es conditio sine qua non ser nacional de un país miembro para adquirir la condición de ciudadano europeo.

La vía opcional que se ha ofrecido por una parte de la doctrina, para incorporar a los residentes no nacionales de un país miembro como ciudadano europeo es el vínculo, el arraigo. Bastida basa ese arraigo en los siguientes criterios:

- La incorporación como vínculo relevante el criterio de residencia permanente

- La lealtad de los ciudadanos hacia su Estado y su comunidad.

Sin embargo, se ha mantenido como criterio necesario para adquirir la ciudadanía europea pertenecer a un país miembro, es decir, ser nacional del mismo, excluyendo a los residentes de terceros países, aunque dicha

${ }^{20}$ Véase Liñán Nogueras, D.J. (2013). «La Ciudadanía Europea: Una Cuestión Abierta», Uned. Teoría y Realidad Constitucional, $\mathrm{n}^{\circ}$ 32, pp. 357-372. Di MAIO, C. Y Tomás, A. (2018). «La ciudadanía europea ante el reto de la unidad política: ¿mero estatuto de libertades o motor para una sólida integración de la Unión Europea?» Revista Derecho del Estado n. ${ }^{\circ}$ 40, enero-junio, pp. 181-20.

${ }^{21}$ Fraile Ortiz, M. (2012). «La ciudadanía europea (entre paréntesis)», en Aláez Corral, B., El pueblo del Estado. Nacionalidad y ciudadanía en el Estado constitucional-democrático, Fundamentos: cuadernos monográficos de teoría del estado, derecho público e historia constitucional, nº 7 , p. 312. 
residencia sea de larga duración y haya creado un vínculo con dicho país, incluso a veces mayor que nacionales que viven fuera de su país de origen.

Otro aspecto importante es la adquisición y pérdida de la ciudadanía europea, la condición, se adquiere siendo nacional de un país miembro de la UE, sin olvidar lo que desarrolla por ley cada país miembro, sin embargo, en lo que habría que centrarse es en la pérdida de esa condición. A grandes rasgos no parece complejo, cuando alguno de los países deje de pertenecer a la UE. Aunque también hay que tener en cuenta la legislación de cada país miembro, lo que da un margen muy amplio de posibilidades, hasta 27 posibles diferentes causas, como ha interpretado en varias ocasiones el TJUE «la determinación de los modos de adquisición y pérdida de la nacionalidad es, de conformidad con el Derecho Internacional, complementaria de cada Estado $(\ldots) »^{22}$.

Dicha jurisprudencia deja abierta otra puerta, cada Estado miembro puede, según su desarrollo legislativo, decidir qué nacionales son considerados ciudadanos europeos, lo que conllevaría según los criterios de cada Estado a una situación discriminatoria, por lo que, habría que fijar criterios mínimos comunes a todos los países ${ }^{23}$. El primer paso que se llevó a cabo, para establecer dichos criterios, fue la aprobación en 1997 del Convenio sobre nacionalidad, pero por ejemplo España no lo ha ratificado, entre otros, por lo que resulta difícil homogeneizar dichos criterios.

El problema continúa situándose en la consideración del concepto de ciudadano-nacional. La ciudadanía entendida como la capacidad de participación en la gestión de los asuntos públicos, siendo únicamente el ciudadano-nacional el que tiene derecho a ser representante y representado, pudiendo, por lo tanto, presentarse como candidato a las elecciones europeas, nacionales y autonómicas, así como participar ejerciendo el derecho de sufragio activo en las mismas ${ }^{24}$. Derechos que serán limitados a los extranjeros de terceros países, pero también, aunque en menor medida a los extranjeros de países miembros. En 1992, hubo un avance, permitiendo a través de la modificación del art. 13.2 de la Constitución española, que los extranjeros de países miembros de la UE puedan ejercer no sólo el derecho de sufragio activo sino también el derecho de sufragio pasivo, no en las elecciones generales, sino en las elecciones municipales. Para que los extranjeros en un país

${ }^{22}$ Al respecto, entre otros casos, STJUE Micheletti, apartado 10, Mesbah apartado 29, Zhu y Chen apartado 37, etc.

${ }^{23}$ Cfr. Fraile Ortiz, M. (2012). El significado de la ciudadanía europea, op.cit., p.178.

${ }^{24}$ Durán Ayago, A. (2015). «Ciudadanía democrática vs identidad nacional: los derechos políticos de los extranjeros en España», Barataria. Revista Castellano- Manchega de Ciencias Sociales, nº19, pp.93-94. 
puedan ejercer este derecho, dependerán de los criterios de reciprocidad que hayan acordado entre los diferentes países, y según lo establecido en los tratados y en la ley. España solo ha firmado Acuerdos con 12 países para que pueda ser ejercido el derecho de sufragio pasivo por los extranjeros de terceros países.

Para participar en las elecciones ${ }^{25}$, los ciudadanos de la UE deberán solicitar su inclusión en el censo electoral del país de residencia, presentando los mismos documentos que los electores nacionales. Asimismo, atenderá que, si en un país miembro se fija un tiempo mínimo de residencia para poder participar del derecho de sufragio activo o pasivo, se entenderá que los ciudadanos de la UE de otro país miembro también deben cumplir dicho requisito ${ }^{26}$.

Según lo que establece la Directiva 94/80/CE del Consejo, de 19 de diciembre de 1994, por la que se fijan las modalidades de ejercicio del derecho de sufragio activo y pasivo en las elecciones municipales por parte de los ciudadanos de la Unión residentes en un Estado miembro del que no sean nacionales, los países de la UE podrán denegar a la ciudadanía de la UE el derecho de sufragio pasivo si:

- Han sido desposeídos del derecho de sufragio pasivo en virtud de la legislación de su país de la UE de origen como consecuencia de una decisión individual en materia civil o penal.

- No pueden presentar una declaración de nacionalidad y residencia u otros documentos que certifiquen su identidad, si se la solicitan.

Los países de la UE podrán decidir que la condición de cargo municipal elegido en el país de residencia es incompatible con funciones ejercidas en otros países UE.

Además de reconocer este derecho de participación en las elecciones al Parlamento europeo y municipal en el estado miembro de residencia, es decir, derecho de sufragio activo y pasivo, la condición de ciudadano europeo conlleva el reconocimiento:

${ }^{25}$ El art. 20 del Tratado de Funcionamiento de la Unión Europea (1985) reconoce «el derecho de sufragio activo y pasivo en las elecciones al Parlamento Europeo y en las elecciones municipales del Estado miembro en el que residan, en las mismas condiciones que los nacionales de dicho Estado»».

${ }_{26}$ También es interesante ver la Directiva 2004/38/CE del Parlamento Europeo y del Consejo de 29 de abril de 2004 relativa al derecho de los ciudadanos de la Unión y de los miembros de sus familias a circular y residir libremente en el territorio de los Estados miembros por la que se modifica el Reglamento (CEE) $n^{\circ} 1612 / 68$ y se derogan las Directivas 64/221/CEE, 68/360/CEE, 72/194/CEE, 73/148/CEE, 75/34/CEE, 75/35/CEE, 90/364/CEE, 90/365/CEE y 93/96/CEE. 
- Del derecho a circular y residir libremente el territorio de los Estados miembros ${ }^{27}$.

- Acceso a la protección diplomática y consular.

- Derecho de petición ante el Parlamento europeo.

- Posibilidad de recurrir ante el Defensor del Pueblo.

- Derecho a una buena Administración y tener acceso a documentos de instituciones y organismos de la Unión.

En el Consejo Europeo de Tampere se prestó especial atención a la situación de aquellos ciudadanos de terceros países que llevaban mucho tiempo residiendo en un país europeo de manera legal y continuada. En 1999 se llevó a cabo una propuesta, sobre el estatuto de los residentes de larga duración, para que una vez que consigan el estatuto de residente de larga duración se les reconozcan una serie de prestaciones similares a la de los nacionales en materia de:

- Condiciones de acceso a un empleo asalariado y a una actividad por cuenta propia, así como condiciones de empleo y trabajo (descanso semanal, normas de higiene, vacaciones anuales, salario, condiciones de despido).

- Educación y formación profesional, reconocimiento de títulos ${ }^{28}$.

- Protección social (subsidios familiares, pensiones de jubilación) y asistencia sanitaria.

- Asistencia social (renta mínima, pensiones mínimas, ayuda médica gratuita).

- Ventajas sociales y fiscales, acceso a bienes y servicios.

- Libertad de asociación y afiliación, y participación en organizaciones de trabajadores o patronos.

- Libre acceso al conjunto del territorio del Estado miembro concerniente.

En cuanto al reconocimiento del derecho de sufragio activo y pasivo a los inmigrantes nacionales de terceros países, es decir, a los ciudadanos no

27 Ver sobre este tema Vidal Fueyo, C. (2006).» La Sentencia del Tribunal Constitucional 72/2005, de 4 de Abril, en materia de Libertad de entrada y residencia de los extranjeros en España», Teoría y Realidad Constitucional, UNED, nº18, pp. 429-441.

${ }_{28}$ Véase Unión Europea. Comunicación de la Comisión al Parlamento europeo, al Consejo, al Comité económico y social europeo y al Comité de las Regiones (2017). «Reforzar la identidad europea mediante la Educación y la Cultura» IN

https://eur-lex.europa.eu/legal-content/ES/TXT/?uri=COM\%3A2017\%3A673\%3AF 
miembros de la Unión Europea, hay que señalar que España presentó en 2005 una Proposición de Ley Orgánica para el reconocimiento del derecho de sufragio activo y pasivo a los ciudadanos extranjeros en España, pero caducó sin ser debatida.

En 2006 se presentó una nueva proposición no de ley, con modificaciones sobre el texto inicial, en la que se instaba al Gobierno a avanzar en criterios de reciprocidad en el ejercicio de derecho de sufragio activo y pasivo de los extranjeros en España, en los términos que establece el art. 13.2 CE, fue aprobada, pero no se dio cumplimiento a lo previsto en dicha proposición ${ }^{29}$. No fue hasta 2008 cuando se volvió a retomar este debate consiguiendo los votos favorables del Gobierno y del PSOE para «el reconocimiento del derecho de voto en las elecciones municipales a todos los inmigrantes con residencia estable» ${ }^{30}$.

Desde 2008 se han firmado 12 acuerdos con diferentes países ${ }^{31}$ con el fin de reconocer a los nacionales de los estados firmantes el derecho de voto en las elecciones municipales. Se establecen como requisitos indispensables: ser mayor de 18 años, tener permiso de residencia, haber residido legal e interrumpidamente en España durante 3 años y deberán estar domiciliados en el municipio en el que les corresponda votar y figurar inscritos en su Padrón Municipal.

En la Constitución española el derecho de participación política viene reconocido en el artículo 23, en el que los ciudadanos tienen derecho a participar en asuntos públicos, directamente o por medio de representantes, libremente elegidos en elecciones periódicas por sufragio universal ${ }^{32}$. Este artículo se complementa con el artículo 13.2 en el que se establece que «solamente los españoles serán titulares de los derechos reconocidos en el artículo 23 , salvo lo que, atendiendo a criterios de reciprocidad, pueda establecerse

${ }^{29}$ Carrasco Durán, M. (2010). El derecho de voto de los extranjeros en las elecciones municipales. Nuevas realidades, Fundación Democracia y Gobierno Local, p. 148.

30 Véase Lucas Martín, J. De y otros (2008). Los derechos de participación como elemento de integración de los inmigrantes, Fundación BBVA, Madrid, pp. 46 y ss, LuCAs Martín, J. De (2009). «Inmigración, diversidad cultural, reconocimiento político, Revista de Sociología, Papers, $\mathrm{n}^{\circ}$ 94, pp. 11-27.

${ }^{31}$ Convenio de reciprocidad en el ejercicio de sufragio activo: Bolivia, Chile, Colombia, Ecuador, Islandia, Noruega, Nueva Zelanda, Paraguay, Perú, Cabo verde, Rusia y Marruecos. https://elpais.com/elpais/2011/01/25/actualidad/1295947035_850215.html $10 / 03 / 2018$.

32 Véase sobre este tema, Rodríguez Ruiz, B. (2015). «Las dos caras de la ciudadanía moderna: entre la nacionalidad y el estatus participativo», Revista Europea de Derechos fundamentales, primer semestre, p. 34, en el que hace referencia al significado de «ciudadanos» del artículo 23 en un sentido estrictamente formal, solo a los que ostentan la nacionalidad española. 
por tratado o por ley para el derecho al sufragio activo y pasivo en las elecciones municipales».

Así, en la actualidad, este derecho se reconoce también a los residentes en España con nacionalidad de otros países de la Unión Europea. En cuanto a los nacionales extracomunitarios, en España, podrán votar en las elecciones municipales siempre que hayan firmado un tratado internacional basado en el principio de reciprocidad con el país de origen, es decir, gozarán del derecho de sufragio todos aquellos nacionales originarios de países con los que España tenga suscrito tratado internacional, en virtud del principio de reciprocidad, entendiendo dicho principio «como cualquier sistema electoral que pueda ser considerado democrático y permita el ejercicio del derecho de sufragio a los residentes españoles, satisface dicho requisito de reciprocidad $»^{33}$, entendiendo, por lo tanto, que no se daría dicha reciprocidad si el Estado contraparte exigiera a los españoles un periodo de residencia sustancialmente diferente al requerido por España. ${ }^{34}$

Aunque realmente no se han celebrado muchos tratados con España que reconozcan a los extranjeros el derecho de sufragio en las elecciones municipales, pues la mayoría de los Tratados no reconocen ese derecho directamente, pues se remiten a lo que se disponga en acuerdos complementarios. ${ }^{35}$

En el caso de Argentina, Colombia, Uruguay o Venezuela existen Tratados Generales de Cooperación y Amistad en los que se prevé que los nacionales de uno y otro Estado puedan votar en las elecciones municipales del Estado de residencia y del que no sean nacionales, conforme a las normas que establezcan en un Acuerdo complementario y ateniéndose a los respectivos regímenes constitucionales y legales. Otros Tratados, sin embargo, solo se remiten a sus respectivas legislaciones, como es el caso de Chile en el Tratado firmado con España sobre Cooperación y Amistad, aunque hay que destacar el Acuerdo específico sobre el reconocimiento del derecho a votar en las elecciones municipales a los nacionales noruegos en España y a los españoles en Noruega (1990), como se detalla en el Informe del Consejo de Estado. Todo ello fiel reflejo de la poca participación de los extranjeros residentes en nuestro país en las elecciones municipales.

Aunque viene regulado en la Ley Orgánica del Régimen Electoral General, en el artículo 176, en el que establece que «... gozan del derecho de sufragio activo en las elecciones municipales los residentes extranjeros en

33 Santolaya, P. y Revenga Sánchez, M. (2006). Nacionalidad, extranjería y derecho de sufragio, Foro inmigración y Ciudadanía, 13, CEPC, Madrid, pp.11-82.

${ }^{34}$ Cfr. Carrasco Durán, M. (2010). El derecho de voto de los extranjeros en las elecciones municipales, op.cit., pp. 149-150.

${ }^{35}$ Llorente, R., Alsina, L., et al. (2009). Informe del Consejo de Estado sobre las propuestas de modificación del Régimen Electoral General, pp. 39-40. 
España cuyos respectivos países permitan el voto a los españoles en dichas elecciones, en los términos de un tratado», matizando a continuación los requisitos necesarios para poder ejercer dicho derecho sin haber adquirido la nacionalidad española, como hemos visto anteriormente.

También se reconoce en el art.177.1. de la LOREG del derecho de sufragio pasivo, en análogas condiciones a las previstas en el art. 176, se exige que el Estado extranjero otorgue a los ciudadanos españoles el derecho de sufragio pasivo en sus elecciones municipales y que se haga, precisamente, en los términos de su tratado, además de precisar que no hayan sido desposeídos del derecho de sufragio pasivo en su Estado de origen.

El reconocimiento del derecho de sufragio a los extranjeros residentes de larga duración, aunque sólo sea en las elecciones municipales ${ }^{36}$, puede considerarse una cuestión de estricta justicia, teniendo en cuenta que contribuyen al desarrollo de su comunidad de residencia, como establece el Informe del Consejo de Estado. Y es que, el reconocimiento de dicho derecho supone una garantía para la integración social de los inmigrantes, incorporando a los extranjeros en la sociedad como miembros de pleno derecho. También se considera como una medida de integración de los extranjeros porque obliga a los partidos políticos, en los procesos electorales, a tener en cuenta las necesidades de esa parte de la población, buscando la manera de dar respuesta a los problemas que les plantean.

Dada la importancia del reconocimiento de dicho derecho a los extranjeros, supone una contradicción y un obstáculo importante que vaya unido al principio de reciprocidad, pues según establece el artículo 13.2 de la CE y la Ley Orgánica 04/2000 de 11 de enero, de los derechos y libertades de los extranjeros en España y su integración social, en su art. 6, sólo se reconoce el derecho de sufragio activo y pasivo a los extranjeros de terceros países cuando se establezca por ley o por tratado con criterios recíprocos y, a tenor de lo que interpreta el TC en su STC 132/1998 «El principio de reciprocidad tiene un significado jurídico preciso, consistente en que un poder público condiciona su actuación a que otro poder público, distinto y ajeno al primero que actúe de la misma manera que él, so pena de que ambos se nieguen reciprocamente lo que a los dos interesa, permaneciendo inactivos o actuando cada uno por su cuenta».

Por lo tanto, como se especifica en la CE y en la legislación estatal, es requisito imprescindible, basarlo en el principio de reciprocidad, siendo más restrictiva la ley que la $\mathrm{CE}$, pues el art. 13.2 solo establece el reconocimiento

36 Véase con más profundidad Arnaldo Alcubilla, E. Y Rodríguez-DrincourT Álvarez, J., (1998). «Una revisión crítica del derecho de sufragio de los extranjeros en las elecciones locales ante la primera aplicación en España», Revista de las Cortes Generales, 45 , pp. 205 y ss. 
de ese derecho en ambos países, siendo objeto mismo del acuerdo, sin embargo la ley de extranjería, establece que deben incluirse las condiciones para su ejercicio debiendo ser iguales o similares en ambos países para poder reconocerse el sufragio a ambos países por igual, por lo que se entiende una versión más restrictiva por parte de la ley. Lo que nos lleva a cuestionarnos si el reconocimiento del derecho de participación política se basa en dicho principio, para que se produzca un intercambio de favores entre Estados o se busca la consideración de ciudadano a toda persona que reside en un Estado.

En España, la política de integración es en su mayor parte responsabilidad de los municipios y de las Comunidades Autónomas, y, dado que muchos municipios carecen de medios financieros y de infraestructuras suficientes, se encuentran con numerosas dificultades para hacer frente a ella. ${ }^{37}$

Ni la Constitución española, ni la Ley de Base del Régimen Local se refieren a la inmigración específicamente. Solamente hacen referencia a los extranjeros en cuanto a su inscripción en el Padrón. Así la Ley 4/2000, de 11 de enero, sobre Derechos y Libertades de los extranjeros en España y su integración social, sólo hace mención a la actividad que deberían desarrollar los municipios en materia de inscripción de los extranjeros en el padrón de los habitantes y con respecto a su participación en el Consejo Superior de Política de Inmigración.

Sin embargo, aunque no son competencias muy amplias, sí imprescindibles para el pleno desarrollo e integración de los inmigrantes en nuestro país, porque se ocupan de dos aspectos necesarios para que puedan iniciar el reconocimiento de derechos y, por tanto, su integración en España. Las administraciones públicas locales son los servicios que se prestan más cercanos a las personas de origen extranjero y a la que se acude para gestionar el padrón, instrumento esencial para el ejercicio de los derechos fundamentales como la educación o a la sanidad, así como las prestaciones y ayudas sociales, de educación y asistencia sanitaria, que son factores determinantes para favorecer la integración social del inmigrante.

El ámbito local ${ }^{38}$ se ocupará de la entrada y residencia de los inmigrantes y del permiso de residencia, que es requisito necesario para poder ejercer, si fuera posible, el derecho de sufragio activo.

37 Ortega Giménez, A y Heredia SÁnchez, L.S. (2008). «El derecho al voto de los extranjeros en las elecciones municipales españolas. ¿Integración o interés?», Anuario mexicano de derecho internacional, vol. 8, UNAM, Instituto de Investigaciones científicas, p.594.

${ }_{38}$ Ver sobre este tema, ExPósito, E. (2013). «Participación ciudadana en el gobierno local. Un análisis desde la perspectiva normativa», Monografías de la Revista Aragonesa de la Administración Pública, XIV, pp. 361-401. 
El art. 149.1.2 de la CE establece que es competencia exclusiva del Estado la materia de «Nacionalidad, inmigración, emigración, extranjería y derecho de asilo», sin embargo, las CCAA han ido reforzando las competencias en relación a las políticas de integración no por vía directa, sino a través del art. 148.1.20 asistencia social, art. 148.1.21 sanidad, en cuanto a la ejecución de los servicios de la Seguridad Social (art. 149.1.17) y del derecho a la educación art. 149.1.30 sobre « la regulación de las condiciones de obtención, expedición y homologación de títulos académicos y profesionales y normas básicas para el desarrollo del derecho a la educación regulado en el art.27 de la CE».

El problema de la ejecución de dichas materias es que cada Comunidad Autónoma dedica diferentes partidas presupuestarias a la asistencia social y a la ejecución sanitaria, por lo que se verán, dependiendo de la Comunidad Autónoma, más beneficiados en unas Comunidades que en otras, existiendo un desequilibrio en el reconocimiento de derechos y prestaciones sociales dependiendo del territorio español en el que se encuentren.

\section{IV.TITULARIDAD DE LOS DERECHOS Y LIBERTADES-LOS DERECHOS DE LOS EXTRANJEROS}

El art. 10.1 de la Constitución española establece que «la dignidad de la persona, los derechos inviolables que le son inherentes, el libre desarrollo de la personalidad, el respeto a la ley y a los derechos de los demás son fundamento del orden político y de la paz social». Por lo tanto, el desarrollo de la personalidad es el único requisito para alcanzar la dignidad, y por consiguiente el disfrute de la capacidad jurídica pareja a él, que vendría a identificarse con la capacidad para ser titular de los derechos inviolables que le son inherentes. $^{39}$

Sin embargo, el ordenamiento jurídico ha basado la titularidad de los derechos en ciertos requisitos discriminatorios, por ejemplo, la nacionalidad, el art. 1.2 de la CE reconoce la soberanía al pueblo español, por lo tanto, restringe la soberanía sólo a los nacionales.

El pleno reconocimiento de todos los derechos vendría restringido por ciertos criterios o condiciones a la hora de ejercerlos, por ejemplo, la mayoría de edad, la nacionalidad, etc. Sin embargo, la STC 197/1991 FJ 4º, considera a los menores como titulares de los derechos y libertades públicas, a pesar de que éstos no puedan ejecutar por sí solos todo su contenido durante el amplio periodo de la minoría de edad. Y en el caso de los extranjeros se pueden

39 Bastida Freijedo, F., ET AL. (2004). Teoría General de los derechos fundamentales en la Constitución española de 1978, Tecnos, Madrid, p.84. 
ampliar los derechos por la ley y los tratados ${ }^{40}$. Por lo tanto, a lo que hay que remitirse no es a la titularidad de los derechos, sino al ejercicio de los derechos, es decir, si ha alcanzado la capacidad de obrar, pues con carácter general una persona por el hecho de serlo, además de titular posee dicha capacidad para ejercer los derechos como señala Bastida ${ }^{41}$, por lo que se entendería que se restringe dicha capacidad en determinados momentos sujeta a ciertos criterios personales o temporales.

En el caso de los criterios de nacionalidad, se han ido modificando y las restricciones que se imponían se han ido actualizando. Por ejemplo, la Constitución española modificó el art. 13.2 como ya hemos visto, así como la aprobación de la respectiva legislación española y europea. Además del Tratado de Maastricht, en 2003 el Dictamen Económico y Social Europeo sobre la incorporación de la UE solicitó que se otorgara la ciudadanía no sólo a los nacionales de los Estados miembros sino también a las personas que residan de manera estable en la UE, es decir, que se reconozca el derecho de sufragio activo y pasivo al Parlamento Europeo y también a las elecciones municipales. La Comisión de Venecia, en el informe sobre la eliminación de restricciones al derecho a voto en las elecciones generales, al menos en las elecciones municipales de los extranjeros residentes, señala que es una cuestión de auténtica justicia, teniendo en cuenta que contribuyen al desarrollo de su comunidad de residencia.

En la STC 107/1984, el Tribunal clasificó los derechos de los no nacionales en tres categorías:

1. Aquellos que pertenecen a la persona en cuanto tal y que son imprescindibles (derivan de la dignidad de la persona, de la garantía de esta, por lo que corresponden por igual a españoles y extranjeros).

2. Los derechos políticos, que como ya hemos visto, salvo excepciones, solo se reconocen a los ciudadanos españoles.

3. Los demás derechos, de los que serán titulares los no nacionales en la medida y términos en los que los reconozcan los Tratados internacionales o las leyes, será en este aspecto donde se reconoce cierta discriminación, la diferencia de trato entre españoles y extranjeros en cuanto al ejercicio de dichos derechos ${ }^{42}$.

${ }^{40}$ Véase en relación con este tema Mangas Martín, A. (2005). «La familia extranjera del ciudadano de la Unión. Una inmigración privilegiada», en Álvarez Conde, E. Y PÉREZ Martín, E. (dirs.), Estudios sobre derecho de extranjería, IDP, Madrid, pp.325344.

${ }^{41}$ Cfr. Bastida Freijedo, F., ET AL. (2004). Teoría General de los derechos fundamentales en la Constitución española de 1978, op. cit., pp. 85-86.

${ }^{42}$ Véase en relación con el reconocimiento de derechos a los extranjeros con más profundidad la Sentencia 107/1984, de 23 de noviembre (BOE núm. 305, de 21 de diciem- 
A pesar de que cada vez es mayor el desarrollo legislativo que protege a los extranjeros de terceros países frente al reconocimiento de derechos, es cierto que se basan en condiciones de desigualdad, pues no existen criterios uniformes para todos los países, permitiendo el desarrollo legislativo a cada uno, y los acuerdos basados en el principio de reciprocidad, que excluye en cierta manera, la posibilidad de que los residentes no nacionales de un país dependan de los acuerdos a los que lleguen los gobiernos de los países, cuando debería ser suficiente con ser persona residente para ser titular de todos los derechos por igual a los ciudadanos de dicho país y no usar como criterio de exclusión la nacionalidad para el ejercicio de determinados derechos, pues lo importante sería promover la integración.

En el marco de los acuerdos de Tampere (oct. 1999) la Comisión presenta una sección sobre la integración de los nacionales de terceros países, la noción de ciudadanía cívica, como un conjunto común de derechos y obligaciones básicas (COM (2000) 757 final). De hecho, la Carta de Derechos fundamentales de la Unión Europea se presenta como la principal base y marco de referencia de la ciudadanía cívica de $2000^{43}$. La noción de ciudadanía cívica se inserta como una vía imprescindible para garantizar la integración de los inmigrantes. La definición de integración debe entenderse como «un proceso bidireccional basado en derechos mutuos y obligaciones correspondientes de los ciudadanos de terceros países en situación legal y de la sociedad de acogida, que permite la plena participación de los inmigrantes. Ello supone, por un lado, que la sociedad de acogida tiene la responsabilidad de garantizar los derechos formales de los inmigrantes de tal manera que estas personas tengan la posibilidad de participar en la vida económica, social, cultural y civil; $y$, por otro lado, que los inmigrantes deben respetar las normas y los valores fundamentales de la sociedad de acogida y participar activamente en el proceso de integración, sin ello tener que renunciar a su propia identidad» ${ }^{44}$.

Se plantean como los elementos necesarios para elaborar las políticas de integración: la educación y los conocimientos lingüísticos, la integración en el mercado laboral, los problemas urbanos y de vivienda, los servicios sanitarios y sociales, el entorno social y cultural, la nacionalidad, la ciudadanía

bre de 1984) sobre Autorización de residencia en España exigida a los extranjeros como requisito para acceder a un puesto de trabajo.

43 Zapata-Barrero, R. (2004). Una nueva «filosofía» de la UE: tradición versus innovación en la propuesta de ciudadanía cívica, II Seminario inmigración y Europa. Cinco años después de Tampere, p.63.

${ }^{44}$ COM (2003) 336 final, de 3 de junio, Comunicación de la Comisión al Consejo, al Parlamento Europeo, al Comité Económico y Social Europeo y al Comité de las Regiones, sobre inmigración, integración y empleo. (Sección 3.1). 
cívica y el respeto por la diversidad (sección 3.3) y para la consecución de dicha integración mediante estas políticas. La Carta de Derechos Fundamentales establece un marco básico de reconocimiento de derechos a la ciudadanía cívica, que se reconoce por su carácter universal y otros derivados de los derechos de los ciudadanos de la Unión ${ }^{45}$.

Lo que plantea la Comisión es reconocer a los inmigrantes la condición de ciudadanía cívica, pues considera que sería muy beneficioso para el proceso de integración en la sociedad y para la adquisición de la nacionalidad. Se trataría de un primer paso, para que las personas residentes de larga duración en un país miembro de la Unión Europea puedan posteriormente alcanzar la ciudadanía europea. Basándose en una definición orientada en la igualdad jurídica «supone la expresión más acabada de la voluntad de la UE de hacer real y efectivo, de forma progresiva y creciente, el derecho indivisible y universal de la igualdad de todas las personas ante la ley» (sección 2.3) ${ }^{46}$ basándose en la igualdad material que defiende como nuevo criterio de atribución de la ciudadanía la residencia permanente.

En la Resolución que el CESE dirigió a la Convención Europea manifestó que «una Comunidad no puede tener en su seno a una parte de miembros en situación de exclusión respecto a los derechos políticos y demás derechos que sí tienen los extranjeros que sean nacionales de los Estados miembros.» Por lo que se apuesta por una ciudadanía europea plural, inclusiva y participativa en base al principio de igualdad de todas las personas ante la ley.

Aunque el Tribunal de Justicia de la Unión Europea ha declarado que un ciudadano de la Unión sólo puede reclamar la igualdad de trato respecto de los nacionales del Estado miembro de acogida, en materia de prestaciones sociales, si su estancia en el territorio cumple con determinados requisitos (medios económicos suficientes y seguro médico). Si no cumplen con dichos requisitos, no tienen derecho a beneficios no contributivos para los inactivos y es que la Directiva 2004/38 es la ley europea que establece las condiciones de ejercicio previstas en el art. 21 del TFUE y exige; o bien tener un trabajo, o tener recursos suficientes y un seguro médico para que ningún ciudadano sea una carga para el Estado de acogida ${ }^{47}$ (aunque hay ciertas adaptaciones

${ }^{45}$ La libertad de circulación y de residencia, el derecho de trabajar, de establecerse o de prestar servicios, el derecho a ser elector y elegible en las elecciones del Parlamento Europeo y en las elecciones municipales, el derecho a la protección diplomática y consular y el derecho de petición, el derecho de acceso a los documentos y la no discriminación sobre la base de la nacionalidad.

46 Dictamen 593/2003 del Comité Económico y Social Europeo sobre «La Incorporación de la ciudadanía de la Unión Europea»

${ }^{47}$ Lo que se pretende es impedir que los ciudadanos de la Unión que no ejerzan una actividad económica utilicen el sistema asistencial del Estado miembro de acogida para garantizar su subsistencia. Sentencia TJUE, Dano, pp. 63 y 76. 
para los estudiantes). La Directiva 2004/38, también dispone como requisitos para poder disfrutar de las prestaciones de asistencia social, la adquisición del derecho de residencia permanente que se adquiere a los cinco años de residencia.

\section{V. ¿ES NECESARIA UNA REFORMA CONSTITUCIONAL RESPECTO A LA CIUDADANÍA?}

La necesidad de lograr el respeto universal y efectivo de los derechos y libertades fundamentales de las personas exige «una concepción común de estos derechos y libertades» que permita el cumplimiento de este compromiso. La Declaración Universal de derechos humanos de 1948 garantiza «el reconocimiento de la dignidad intrínseca y de los derechos iguales e inalienables de todos los miembros de la familia humana». Por tanto, si nos basamos en el reconocimiento de que estos derechos son iguales e inalienables a todos los seres humanos, podemos decir que todos los seres humanos son ciudadanos. Todos son titulares de los derechos humanos, todos son libres e iguales, $\mathrm{y}$, por tanto, todos deben ser educados en una cultura de derechos humanos para lograr el respeto mutuo y una convivencia cívica pacífica ${ }^{48}$.

La construcción jurídica de la ciudadanía multinivel, es decir la diferenciación en grados de la ciudadanía dependiendo del ámbito (estatal, autonómico, local) «ha de consistir en poner a disposición del individuo el acceso a grados de ciudadanía adecuados a la intensidad de su presencia y permanencia en la comunidad de la que forma parte ${ }^{49}$. Desde el punto de vista de una sociedad democrática, la nacionalidad y la ciudadanía han de construirse desde la sociedad de individuos libres e iguales que se relacionan entre sí, y no desde la pertenencia de una nacionalidad.

Por lo tanto, una propuesta de reforma constitucional sería no reconducir la ciudadanía sólo a la nacionalidad sino también a la vecindad, para poder dotar de plenos derechos de participación política a los residentes de nacionalidad extranjera para permitir un proceso de integración mayor y evitar exclusiones por razones de nacionalidad. El art. 22 del Código civil prevé que «para la concesión de la nacionalidad por residencia se requiere que ésta haya durado 10 años, serán suficientes 5 años para los que hayan obtenido la condición de refugiado, dos años de residencia legal continuada, inmediatamente anterior a la solicitud de la nacionalidad cuando se trate de nacionales de origen de países iberoamericanos, Andorra, Filipinas, Guinea

${ }^{48}$ Souto Paz, J.A. (2010). Educación, democracia y ciudadanía, Dykinson, Madrid, p.24.

49 Bastida Freijedo, F., ET AL. (2004). Teoría General de los derechos fundamentales en la Constitución española de 1978, Tecnos, Madrid, p.27. 
Ecuatorial o Portugal o sefardies» o de un año para "el nacido fuera de España de padre o madre, abuelo o abuela, que originariamente hubieran sido españoles».

La adquisición de nacionalidad lleva consigo la plenitud de derechos de ciudadanía. Lo que se propone es democratizar el acceso a la nacionalidad, cuando se trate de una condición para el disfrute de la ciudadanía, reduciendo plazos y requisitos, pero también, evitando el reconocimiento de derechos solo por la adquisición de la nacionalidad. Es decir, que los residentes de larga duración, puedan ser sujetos de reconocimiento de derechos, en concreto del derecho de sufragio activo y pasivo ${ }^{50}$. Para lo que se plantearía la modificación del art. 13.2, incluyendo a los residentes de larga duración y eliminando la necesidad de basarse en el principio de reciprocidad, lo que permitiría una mayor integración de los nacionales de otros países en el Estado de residencia e incluso favorecería la expresión de igualdad jurídica y del pluralismo participativo necesarios en un Estado democrático.

En cuanto a la propuesta de modificación del principio de reciprocidad ${ }^{51}$, si tenemos en cuenta la importancia del reconocimiento de este derecho a los extranjeros, vincularlo a dicho principio supone una contradicción y un obstáculo importante ${ }^{52}$. Según establece el artículo 13.2 de la Constitución española y el art. 6 de la Ley Orgánica 04/2000 de 11 de enero, de los derechos y libertades de los extranjeros en España y su integración social, sólo se reconoce el derecho de sufragio activo y pasivo a los extranjeros de terceros países cuando se establezca por ley o por tratado con criterios recíprocos y, a tenor de lo que interpreta el TC en su STC 132/1998 «El principio de reciprocidad tiene un significado jurídico preciso, consistente en que un poder público condiciona su actuación a que otro poder público, distinto y ajeno al primero que actúe de la misma manera que él, so pena de que ambos se nieguen recíprocamente lo que a los dos interesa, permaneciendo inactivos o actuando cada uno por su cuenta».

${ }^{50}$ Como señala la profesora Guichot, la participación democrática que se pide es la de todos los ciudadanos y ciudadanas. No la de una minoría...quienes no tengan el reconocimiento de la misma capacidad política que sus conciudadanos/as no son realmente libre porque solo podemos evitar la dominación si no dejamos las decisiones en manos de una minoría, ver Guichot, R. (2013). «Participación, ciudadanía activa y educación», Teoría de la Educación, Revista Interuniversitaria, 25, 2, pp. 32-33.

${ }^{51}$ Cfr. Rodríguez Ruiz, B. (2015). «Las dos caras de la ciudadanía moderna: entre la nacionalidad y el estatus participativo», op.cit., p. 34.

52 Ver sobre este tema AJA, E. Y MoYA, D. (2008). «El derecho de sufragio de los extranjeros residentes», en Aja Fernández, E., Arango Vila-Belda, J. Y Oliver Alonso, J. (coords.). La inmigración en la encrucijada. Anuario de la inmigración en España, Bellaterra, Barcelona, pp. 64-81. 


\section{BIBLIOGRAFIA}

Aja, E., Moya, D. (2008). «El derecho de sufragio de los extranjeros residentes», en E. Aja Fernández, E, Arango Vila-Belda, J. y Oliver Alonso, J. (coords.), La inmigración en la encrucijada. Anuario de la inmigración en España, Bellaterra, Barcelona.

Aláez Corral, B. (2005). Nacionalidad y ciudadanía: una aproximación históricafuncional, vol. 6, Historia Constitucional: Revista electrónica: http://hc.rediris. es/06/index.html

Arnaldo Alcubilla, E. y Rodríguez-Drincourt Álvarez, J. (1998). «Una revisión crítica del derecho de sufragio de los extranjeros en las elecciones locales ante la primera aplicación en España», en Revista de las Cortes Generales, 45, p. 205 y ss.

Bastida FreiJedo, F., et al. (2004). Teoría General de los derechos fundamentales en la Constitución española de 1978, Tecnos, Madrid.

Biglino Campos, P. (1995). «Ciudadanía europea y legitimidad democrática», Revista de Estudios Europeos, $\mathrm{n}^{\circ} 9$.

Carrasco Durán, M. (2010), El derecho de voto de los extranjeros en las elecciones municipales. Nuevas realidades, Fundación Democracia y Gobierno Local. http://repositorio.gobiernolocal.es/xmlui/bitstream/handle/10873/658/07\%20 QDL\%2022\%20ES-carrasco..pdf? sequence=1\&isAllowed $=y$

Di Maio, C y Tomás, A. (2018), La Ciudadanía Europea ante el Reto de la Unidad Política: ¿Mero Estatuto de libertades o motor para una sólida integración de la Unión Europea?, Revista Derecho Del Estado no 40, Enero-Junio.

Durán Ayago, A. (2015). «Ciudadanía democrática vs identidad nacional: los derechos políticos de los extranjeros en España», Barataria. Revista CastellanoManchega de Ciencias Sociales, $\mathrm{n}^{\circ} 19$.

Expósito, E. (2013). «Participación ciudadana en el gobierno local. Un análisis desde la perspectiva normativa», Revista Aragonesa de la Administración Pública, XIV.

Fraile Ortiz, M. (2002). El significado de la ciudadanía europea, Centro de Estudios Políticos y Constitucionales, Madrid.

- (2012). «La ciudadanía europea (entre paréntesis)», Fundamentos: cuadernos monográficos de teoría del estado, derecho público e historia constitucional, $\mathrm{n}^{\circ} 7$.

Guichot, R. (2013). «Participación, ciudadanía activa y educación», Teoría de la Educación, Revista Interuniversitaria, 25, 2.

Liñán Nogueras, Diego J. (2013). «La Ciudadanía Europea: Una Cuestión Abierta», Uned. Teoría y Realidad Constitucional, $\mathrm{n}^{\mathrm{o}} 32$.

LuCAS MARTín, J. DE Y OTROS (2008). Los derechos de participación como elemento de integración de los inmigrantes, Fundación BBVA, Madrid.

LuCAS MARTíN, J. DE (2009). «Inmigración, diversidad cultural, reconocimiento político, Revista de Sociología, Papers, $\mathrm{n}^{\circ} 94$.

(2004). «Ciudadanía, extranjería y derechos», en AA.VV., Constitución y derechos fundamentales, Ministerio de la Presidencia, CEPC, Madrid.

Llorente, R., Alsina, L., et al. (2009). Informe del Consejo de Estado sobre las propuestas de modificación del Régimen Electoral General. 
Máız, R. (2005). «Nacionalismos e inmigración en Francia: la Republique une et indivisible y el affaire du foulard», Revista de Estudios políticos (nueva época), $\mathrm{n}^{\mathrm{o}} 129$, Madrid, julio-septiembre.

Mangas Martín, A. (2016). «La Retirada del Reino Unido de la Unión Europea», Foro, Nueva Época, vol. 19, $\mathrm{n}^{\circ} 1$.

(2005). «La familia extranjera del ciudadano de la Unión. Una inmigración privilegiada», Álvarez Conde, E. y PÉrez Martín, E. (dirs.), Estudios sobre derecho de extranjería, IDP, Madrid.

Moreno, L. (2012). «Ciudadanía multinivel», en Ciudadanía y derechos sociales (IV Congreso Internacional de derechos humanos), Vitoria-Gasteiz, Servicio central de publicaciones del Gobierno Vasco.

Ortega Giménez, A y Heredia Sánchez, L.S. (2008). El derecho al voto de los extranjeros en las elecciones municipales españolas. ¿Integración o interés? Anuario mexicano de derecho internacional, vol. 8, UNAM, Instituto de Investigaciones científicas.

PÉrez Vera E. (1996). «Citoyenneté de 1'Union Européenne, nationalité et condition des étrangers», Recuil des Courses. Collected curses of the Hague Academy of International Law, Vol. 261, Martinus Nifhoff Publishers.

RoDRíguez Ruiz, B., «Las dos caras de la ciudadanía moderna: entre la nacionalidad y el estatus participativo», Revista Europea de Derechos fundamentales, primer semestre 2016.

Santolaya, P., y Revenga Sánchez, M. (2006). Nacionalidad, extranjería y derecho de sufragio, Foro inmigración y Ciudadanía, 13, CEPC, Madrid.

Souto Paz, J.A. (2010). Educación, democracia y ciudadanía, Dykinson, Madrid.

Souto Galván, C. (2015). «Hacia un nuevo concepto de ciudadanía», en TuR AusiNA, R. (dir.), Problemas actuales de derecho constitucional en un contexto de crisis, Comares, Madrid.

Vidal Fueyo, C. (2006). «La Sentencia del Tribunal Constitucional 72/2005, de 4 de Abril, en materia de Libertad de entrada y residencia de los extranjeros en España», Teoría y Realidad Constitucional, UNED, $\mathrm{n}^{\circ} 18$.

Villaverde MenéndeZ, I. (2012). «La ciudadanía borrosa: Ciudadanías multinivel», Cuadernos monográficos de teoría del estado, derecho público e historia constitucional, $\mathrm{n}^{\mathrm{o}}$ 7, (Ejemplar dedicado a: El pueblo del Estado. Nacionalidad y Ciudadanía en el Estado Constitucional / coord. por Benito Aláez Corral)

ZAPATA-BARRERo, R. (2004). Una nueva «filosofía» de la UE: tradición versus innovación en la propuesta de ciudadanía cívica, II Seminario Inmigración y Europa. Cinco años después de Tampere, Barcelona, Fundación Cibob.

\section{Documentos Oficiales}

Ley Orgánica 4/2000, de 11 de enero, sobre derechos y libertades de los extranjeros en España y su integración social.

Directiva 94/80/CE del Consejo, de 19 de diciembre de 1994, por la que se fijan las modalidades de ejercicio del derecho de sufragio activo y pasivo en las elecciones 
municipales por parte de los ciudadanos de la Unión residentes en un Estado miembro del que no sean nacionales.

DICTAMEN del Comité Económico y Social Europeo sobre el tema «Incorporación a la ciudadanía de la Unión Europea» (Dictamen de iniciativa). Comité Económico, Social y europeo, SOC/141 «La ciudadanía europea» Bruselas, 14 de mayo de 2003.

Directiva 2004/38 del Parlamento y del Consejo de 29 de abril de 2004. La Directiva 2004/38/CE, del Parlamento Europeo y del Consejo, de 29 de abril de 2004, relativa al derecho de los ciudadanos de la Unión y de los miembros de sus familias a circular y residir libremente en el territorio de los Estados miembros, por la que se modifica el Reglamento (CEE) n. ${ }^{\circ} 1612 / 68$ y se derogan las Directivas 64/221/ CEE, 68/360/CEE, 72/194/CEE, 73/148/CEE, 75/34/CEE, 75/35/CEE, 90/364/ CEE, 90/365/CEE y 93/96/CEE.

\section{WEBGRAFIA}

http://www.mjusticia.gob.es/cs/Satellite/Portal/es/ciudadanos/tramites-gestionespersonales/nacionalidad-residencia, recuperado el 05/03/2018

http://hc.rediris.es/06/index.html, recuperado el 05/03/2018 http://eur-lex.europa.eu/legal-content/ES/TXT/?uri=LEGISSUM\%3A123026, recuperado el 08/03/2018

http://repositorio.gobiernolocal.es/xmlui/bitstream/handle/10873/658/07\%20

QDL $\% 2022 \% 20 E S$-carrasco..pdf? sequence=1\&isAllowed=y, recuperado el $10 / 03 / 2018$

https://www.navascusi.com/wp-content/uploads/2016/07/Brexit_espa\%C3\%B1ol. pdf, recuperado el 13/03/2018

https://europa.eu/european-union/topics/eu-citizenship_es, recuperado el $13 / 03 / 2018$. 


\title{
CIUDADANÍA MULTINIVEL Y SU ENCAJE CONSTITUCIONAL
}

\author{
Multilevel citizenship and ownership
}

\author{
Clara Souto Galván \\ Profesora contratada doctora de Derecho Constitucional \\ Universidad Rey Juan Carlos
}

http://dx.doi.org/10.18543/ed-68(1)-2020pp449-473

\section{Copyright}

Estudios de Deusto es una revista de acceso abierto, lo que significa que es de libre acceso en su integridad. Se permite su lectura, la búsqueda, descarga, distribución y reutilización legal en cualquier tipo de soporte sólo para fines no comerciales, sin la previa autorización del editor o el autor, siempre que la obra original sea debidamente citada y cualquier cambio en el original esté claramente indicado

Estudios de Deusto is an Open Access journal which means that it is free for full access, reading, search, download, distribution, and lawful reuse in any medium only for non-commercial purposes, without prior permission from the Publisher or the author; provided the original work is properly cited and any changes to the original are clearly indicated. 